\title{
Accurate calculation research on scattering characteristics of gap
}

\author{
Jingcheng Zhao ${ }^{1}$, Shuo Yang ${ }^{1, a}$, Xu Gao ${ }^{2}$ and Jianzhong Chai ${ }^{2}$ \\ ${ }^{1}$ BeiHang University, Electronic information engineering college, 100191, Beijing, China \\ ${ }^{2}$ Avic The First Aircraft Institute, Production Department, 710089, Xi an, China
}

\begin{abstract}
For more accurately studying electromagnetic scattering characteristics of slit, the paper proposed a new method of vector cancellation. Firstly, the feasibility of the method is verified by the experimental method, then, this method is used to study the law of the gap electromagnetic scattering with the change of gap width, as well as multi frequency characteristics with the change of incident frequency. Calculation results show that: In the range of about $\pm 30^{\circ}$, the gap induced surface wave scattering increases with the increase of the gap width and increases with the increase of the incident frequency; In the range of grazing incidence $-90^{\circ} \sim-60^{\circ}$ and $60^{\circ} \sim 90^{\circ}$, the scattering curve of gap gradually become flat with the increase of the incident electromagnetic wave frequency.
\end{abstract}

Keywords: vector cancellation; electromagnetic scattering; gap; radar cross section; stealth.

\section{Introduction}

The stealth aircraft has taken effective measures to reduce the main scattering sources such as smooth metal mirror and cavity, and its overall radar cross section is lower than that of the conventional aircraft[1].At this time, the gap as the representative of the discontinuous characteristics of the total scattering will greatly increase the proportion of the total scattering, and even the effect is very obvious in some polarization and attitude angle[2-4].Therefore, it is necessary to study its electromagnetic scattering characteristics in detail[6-9],getting the scattering mechanism[10].

At present, research methods of gap scattering characteristics can be divided into 2 types: anechoic chamber testing and numerical calculation. Literature[17] is the anechoic chamber test, it is concluded that the gap scattering characteristics change with gap width as well as gap spacing; Literature[12] is the numerical method, which is used to study the relationship between the single gap changes with the width and the three gap changes with the distance and the polarization mode; However, in addition to the scattering of the gap, the results of the above two papers also contain the scattering of the carrier itself and the coupling between the edge and the edge of the carrier, which can not fully reflect the accurate results of the scattering of the scattering in each corner. Although anechoic chamber test has advantages in the accuracy and reliability of the results, it need to make lots of samples, high cost, long cycle; numerical calculation method designs short cycle and uses flexibility and conveniently. According to the existing literature [11-16], the main methods to study

\footnotetext{
${ }^{\mathrm{a}}$ Corresponding author : yangshuo152633@163.com
} 
the scattering characteristics are: method of moment (MOM) [11], multilevel fast multipole algorithm (MLFMA) [12-14] or finite difference time domain method (FDTD) [15, 16].

In order to get more precise slit scattering characteristics, taking into account the cost and time, this paper selects single straight gap, through the software FEKO multilevel fast multipole algorithm [19] calculated electromagnetic scattering, which contains the slot of the metal plate and does not contain the crevice in the metal plate, by vector cancellation, getting simple slot scattering characteristic curve. Fist by contrast with the test curves in Literature [17], proving the accuracy of calculation results, then using the calculation method to get the rules that the electromagnetic scattering of single straight silt changes with gap width and incident frequency.

\section{Mathematical statements}

The objective electromagnetic scattering characteristic commonly uses Radar Cross Section (RCS) to mesure. RCS represented by $\sigma$, then the formula (1):

$$
\sigma=\lim _{R \rightarrow \infty} 4 \pi R^{2} \frac{\left|\vec{E}^{s}\right|^{2}}{\left|\vec{E}^{i}\right|^{2}}=\lim _{R \rightarrow \infty} 4 \pi R^{2} \frac{\left|\vec{S}^{s}\right|}{\left|\vec{S}^{i}\right|}
$$

Gaps and other weak scattering targets can not exist independently, they must be attached to a certain type of carrier. For example, slotting in a piece of metal plate, if there is no plate, there is no gap exists, this plate is the gap of the carrier. If the carrier is infinite, there is no coupling phenomenon except for the scattering of the gap itself. In this case, the gap can be equivalent to the surface electromagnetic flow, using the method of moments to solve. But for the actual use and evaluation of the gap target, it must be attached to the finite size of the carrier, in this case, the total scattering can be divided into three parts [4]: (a) the scattering of the gap itself; (b) the scattering of the carrier itself; (c) the coupling between the edge of the carrier and the gap.

In view of this situation, from the published results, the focus of the research work is actually on the reduction of the carrier itself scattering [5]. Edge diffraction itself is a kind of weak scattering source, if the carrier itself is a low scattering, then coupling of the edge and the gap is also very small. According to this feature, a vector cancellation method is used to deal with the results obtained by using the conventional calculation method, getting the scattering results which can reflect the gap itself.

First, using the conventional calculation method to get electric field component of metal plate with gap $\overrightarrow{\mathrm{E}}_{\theta}, \overrightarrow{\mathrm{E}}_{\varphi}$ and electric field component of the metal plate without gap $\overrightarrow{\mathrm{E}}_{\theta 1}, \overrightarrow{\mathrm{E}}_{\varphi 1}$; Then vector cancellation formula is introduced:

$$
\begin{aligned}
\overrightarrow{\mathrm{E}}_{f a r, \theta} & =\overrightarrow{\mathrm{E}}_{\theta}-\overrightarrow{\mathrm{E}}_{\theta 1} \\
\overrightarrow{\mathrm{E}}_{f a r, \varphi} & =\overrightarrow{\mathrm{E}}_{\varphi}-\overrightarrow{\mathrm{E}}_{\varphi 1}
\end{aligned}
$$

Obtaining a new component which does not contain the contribution of the carrier scattering $\overrightarrow{\mathrm{E}}_{\text {far }, \theta}$ and $\overrightarrow{\mathrm{E}}_{f a r, \varphi}$. At last, the RCS value is calculated by the RCS calculation formula:

$$
\sigma=\lim _{R \rightarrow \infty} 4 \pi \frac{\left|R \overrightarrow{\mathrm{E}}_{\theta}^{1}\right|^{2}+\left|R \overrightarrow{\mathrm{E}}_{\varphi}^{1}\right|^{2}}{\left|\overrightarrow{\mathrm{E}}_{i}\right|^{2}}=4 \pi \frac{\left|\overrightarrow{\mathrm{E}}_{f a r, \theta}\right|^{2}+\left|\overrightarrow{\mathrm{E}}_{f a r, \varphi}\right|^{2}}{\left|\overrightarrow{\mathrm{E}}_{i}\right|^{2}}
$$


After elimination, the RCS value contains the contribution of gap itself and Interaction between edge and slit. It should be explained that, due to the weak coupling between the edge and the gap, it can be considered that this data is mainly due to the scattering of the gap.

The method improves the precision of calculating weak scattering target electromagnetic scattering such as silt, etc. removes the restrictions of weak scattering target vector shape, increases the size of the gap of weak scattering target measured, it has a very good practical prospects, especially in the gap analysis, this weak scattering source has important significance on the influence of stealth equipment such as aircraft.

In order to verify the method, this paper selects a single direct gap model in the literature [17], as shown in figure 1 . The model comprises a square metal plate with a single straight slit, a square metal plate without gaps; Slit length is $200 \mathrm{~mm}$, width is $5 \mathrm{~mm}$, the incident frequency is $10 \mathrm{GHz}$, and the choice of horizontal polarization $(\mathrm{HH})$, the RCS value of a square metal plate containing a single straight slit (shown in Figure 2) and the RCS value of a square metal plate without gaps is calculated, by vector cancellation, getting containing gap and scattering characteristic curve of coupling and the gap edge of the carrier, as shown in Figure 3 the solid line.

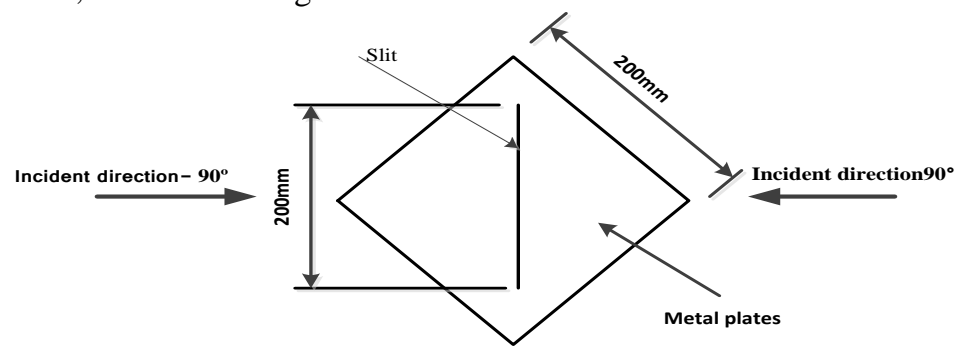

Figure 1. Single gap calculation model of variable width

By Figure 2 and literature [17] contrast can be found: in the incident angle of $-15^{\circ} \sim 15^{\circ}$ range, near the normal incidence, the scattering of the gap itself was drowned out by the carrier of the specular scattering and contrast two pictures, in the angle domain the calculation results and the measured results are consistent; In the range of $-60^{\circ} \sim-15^{\circ}, 15^{\circ} \sim 60^{\circ}$, slit scattering is stronger than the scattering of the carrier, and the waveform of the two figure is the same. The calculated results are basically consistent with the experimental results; In the range of $-90^{\circ} \sim-60^{\circ}, 60^{\circ} \sim 90^{\circ}$, compared with the two images, the calculated results in this angular field are in agreement with the measured results. The reason why the two images are not identical is due to the interference of the background noise. Compared with the measured curve, the MLFMA algorithm is used to calculate the scattering characteristic curve and the measured curve of the metal plate with a single straight slit, which are consistent ; Then using the cancellation method to obtain the scattering characteristic curve with a single straight slit, as shown in Figure 3 the solid line: in the incident angle of $-15^{\circ} \sim 15^{\circ}$ range, near the normal incidence, before the cancellation, the scattering of the gap itself is covered by specular scattering of a carrier, after losing the carrier scattering, this time we can clearly get the real RCS value that gap in this angle domain; In the range of $-60^{\circ} \sim-15^{\circ}, 15^{\circ} \sim 60^{\circ}$, slit scattering is stronger than the scattering of the carrier, the eliminated curve should be close to not eliminated scattering curve, comparing with the solid line and dotted line of Fig. 3, found that the two curves are fit; In the range of $-90^{\circ} \sim-60^{\circ}, 60^{\circ} \sim 90^{\circ}$, Gap induced travelling wave scattering is stronger than the seamless metal plate, interference of the angle domain carrier scattering of the slit is minimal, reflecting in the figure, the solid line and dotted line is basically consistent in the angle domain. At about $\pm 75^{\circ}$, the lower coupling of the solid line and dotted line is caused because the gap and the metal plate edge coupling.

By comparing with the curve of literature [17], it can be known that, the results obtained by this method are reliable, and the accurate scattering curves of the gap in the forward angle domain($30^{\circ} \sim 30^{\circ}$ )are extracted, the gap scattering data is extended to the whole domain, meeting the need of further research. 


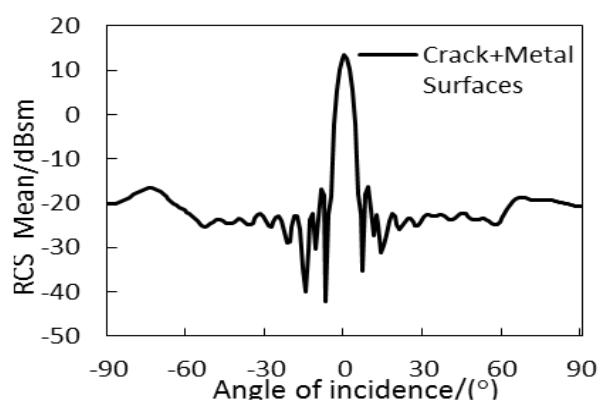

Figure 2. Single slit metal plate RCS (HH polarization)

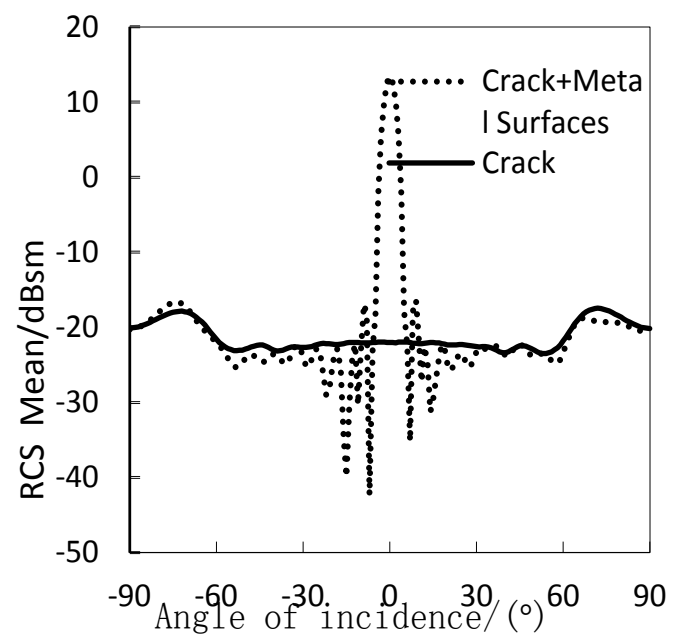

Figure 3. Comparison results of single slit cancellation

The calculation model of single slit scattering characteristic changing with crack width is studied in Figure 1. The incident angle changes at- $90^{\circ} \sim 90^{\circ}$. Gap width is a, selecting a=1, 3, 5, 8, 12, $16 \mathrm{~mm}$. The incident frequency is selected as $10 \mathrm{GHz}$, and the polarization mode is selected for $\mathrm{HH}$ polarization.

In order to study the law of single slit scattering characteristics changing with incident frequency, the computational model selects Figure 1, the incident angle changes in $-90^{\circ} \sim 90^{\circ}$, the gap width is 5mm, The incident frequency is: $6 \mathrm{GHz}$ (XC band), $10 \mathrm{GHz}$ ( $\mathrm{X}$ band), $12 \mathrm{GHz}$ (X band), $15 \mathrm{GHz}$ (Ku band), selecting $\mathrm{HH}$ polarization.

For the convenience of research, the model diagonal length is $\mathrm{L}$, which is defined as: $\mathrm{R}=\mathrm{L} / \lambda$, $\lambda$ is incident electromagnetic wave length; The calculation frequency is10 $\mathrm{GHz}$, corresponding $\mathrm{R}=9$, which in resonance region.

\section{Results and discussion}

\subsection{Scattering characteristics of gap changing with gap width}

Figure 4 shows that under the polarization of $\mathrm{HH}$, calculation results of single slit whose gap width is respectively $1,3,5,8,12,16 \mathrm{~mm}$ on RCS after elimination. 


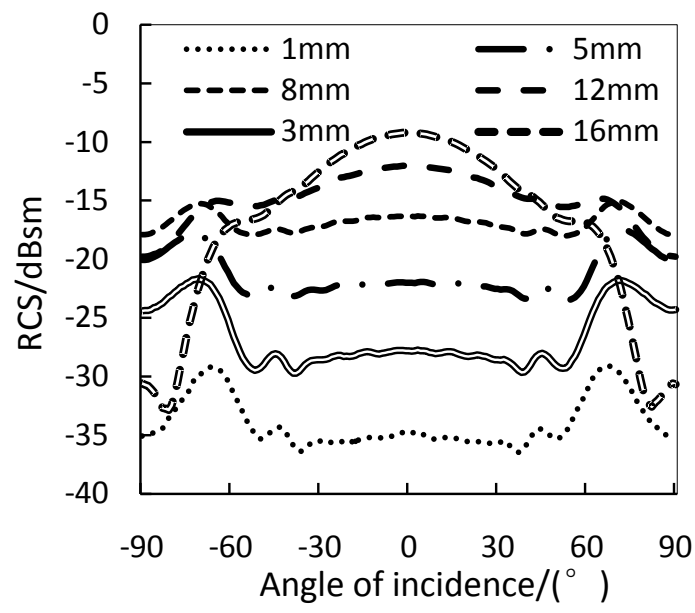

Figure 4. The eliminated single slit RCS (HH polarization)

The distribution characteristics of RCS can be obtained from the figure 4:

(1)When the incident angle is in the range of $-30^{\circ} \sim 30^{\circ}$, using the elimination method can get the value of RCS which gap is at normal incidence.

(2) When the incident angle is in the range of $-60^{\circ} \sim-30^{\circ}, 30^{\circ} \sim 60^{\circ}$, the slit scattering intensity and scattering curve surge with the increase of the angle up and down.

(3) When the incident angle is in the range of- $90^{\circ} \sim-60^{\circ}, 60^{\circ} \sim 90^{\circ}$, electromagnetic scattering in the corner is still strong, scattering curve in the vicinity of $\pm 75^{\circ}$ has the strongest wave.

According to the calculated curve figure 5, we can get RCS mean value of single slit with different width under the polarization of $\mathrm{HH}$, view a single gap width as the variable, drawing the curve graph which the RCS mean changes with the width, as shown in Figure 9, which is used to study the change of RCS with the width of the gap.

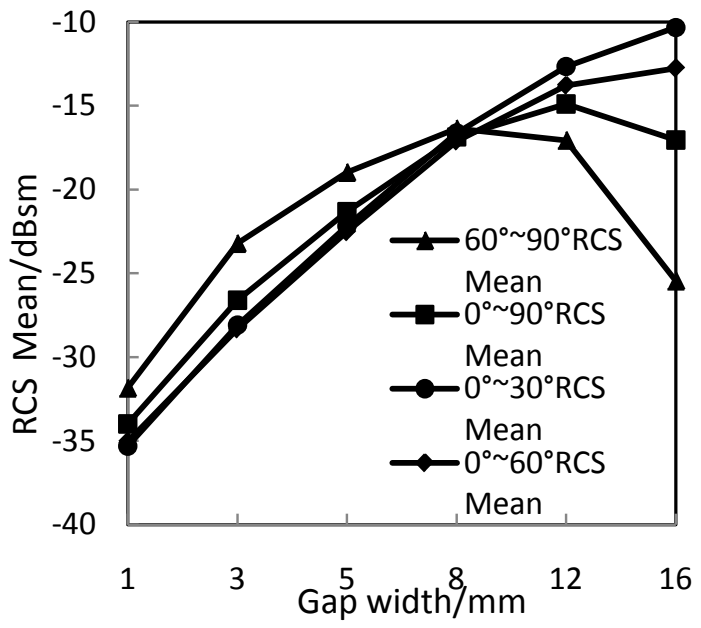

Figure 5. The change law of RCS mean value in different angular fields (HH polarization)

From Figure 5 can be found: gap width increase did not cause the RCS has been increased, but with the increase of the width, the RCS of the gap increased first and then reduce $\left(60^{\circ} \sim 90^{\circ}\right.$ in average); In the $0^{\circ} \sim 90^{\circ}$ angle domain, the overall scattering increased first and then decreased, which is different from the overall scattering increase amplitude tends to be stable in the $0^{\circ} \sim 90^{\circ}$ angle domain in the literature [17],because in the literature [17],it uses mean value subtract, the results did not completely 
eliminate the carrier scattering. In the $0^{\circ} \sim 30^{\circ}$ angle domain, because the interference to eliminate the carrier specular scattering and coupling the angle domain gap with the edge of the carrier is very low, this range can be seen as electromagnetic scattering from slit and scattered gap with the increase of the width increases; In the $0^{\circ} \sim 60^{\circ}$ angle domain, scattered gap increases with the width and growth rate becomes slow, because after vector cancellation, the slit scattering also contains the coupling of the edge and the gap, with the increase of angle domain, slot width, the coupling effect enhancement, so that the gap scattering amplitude is slowing.

In this calculation, because the gap length is constant, analysing the regulation of gap RCS changing with gap width can be understood as an analysis of the law of the gap RCS changing with $\mathrm{a} / \lambda$. For $\mathrm{HH}$ polarization, in the $0^{\circ} \sim 60^{\circ}$ angle domain, gap originated electromagnetic scattering: when a is less than $\lambda$ 's $1 / 4$ (that is $a=1,3,5,8 \mathrm{~mm}$ ) as well as a is more than $\lambda$ 's $1 / 4$ and continue to increase(that is $\mathrm{a}=12,16 \mathrm{~mm}$ ), with the increase of $\mathrm{a}$, the corresponding RCS is increased; For the $60^{\circ} \sim 90^{\circ}$ angle domain gap scattering: when a is less than $\lambda$ 's $1 / 2$, a increase will lead to the rapid growth of gap RCS; When the gap width is close to the wavelength of $1 / 2$, (that is, the gap width of 16 $\mathrm{mm}$ ), slit scattering Instead lower, reflected in figure $5,60^{\circ} \sim 90^{\circ}$ in angle domain $16 \mathrm{~mm}$ wide gap caused by the scattering curve than other curve to be low, because the $16 \mathrm{~mm}$ wide, both sides of the gap caused by the surface waves after the $\lambda / 2$ distance, two surface wave phase difference meets $180^{\circ}$, the sum of the reflected surface amplitude value is smaller.

\subsection{The scattering characteristics of gap varies with incident frequency}

Figure 6 shows the RCS calculation results when the gap varies with the incident frequency.

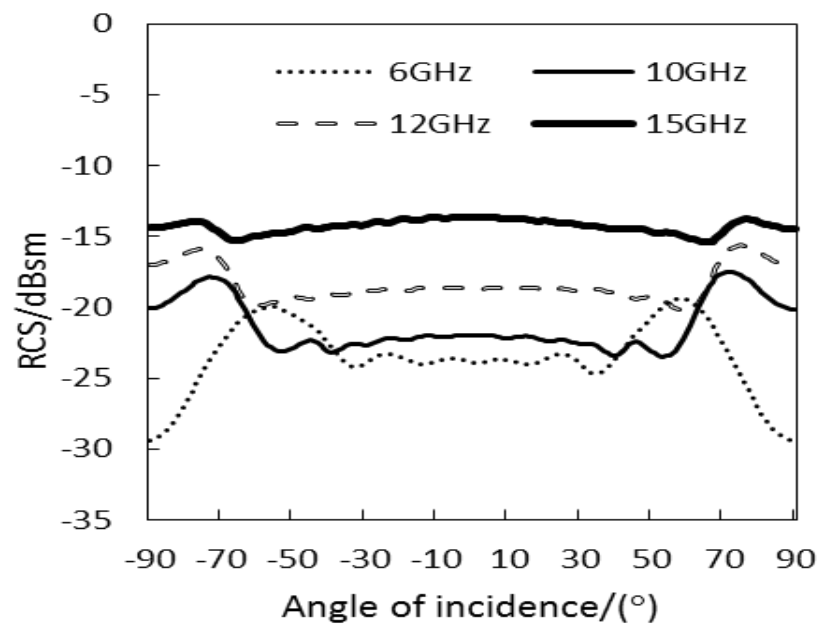

Figure 6. RCS of the single gap varies with the incident frequency (HH polarization)

The following conclusions can be drawn from figure 6:

(1) Frequency from $6 \mathrm{GHz}$ to $15 \mathrm{GHz}$, the spatial distribution of the curve is consistent, which can be divided into 2 parts: grazing incidence of wave scattering caused by the second wave crest area, $50^{\circ} \sim 50^{\circ}$ in the area of the flat area.

(2) Traveling wave scattering caused the second peak is outwordly migration with the increase of the frequency, and the curve gradually become flat. When the incident frequency is $6 \mathrm{GHz}$, the second wave peak appears at about $\pm 60^{\circ}$, and the peak is the maximum; When the incident frequency is 10 $\mathrm{GHz}, 12 \mathrm{GHz}, 15 \mathrm{GHz}$, the peak respectively appears at about $\pm 70^{\circ}, \pm 74^{\circ}, \pm 75^{\circ}$, and gradually narrow. The peak value and the whole curve increase with the increase of frequency.

(3) In the vicinity of $-30^{\circ} \sim 30^{\circ}$, before the cancellation,it is specular scattering of the carrier, after the elimination, the scattering characteristics of the gap itself can be clearly obtained by the method. 
Table 1. The arithmetic mean value of each angle field at different incidence frequency

\begin{tabular}{|c|c|c|c|c|}
\hline Freq & $-90^{\circ} \sim-60^{\circ}$ & $-60^{\circ} \sim-10^{\circ}$ & $-10^{\circ} \sim 0^{\circ}$ & $-90^{\circ} \sim 0^{\circ}$ \\
\hline $6 \mathrm{GHz}$ & -24.779 & -22.484 & -23.726 & -23.426 \\
\hline $10 \mathrm{GHz}$ & -19.124 & -22.521 & -22.026 & -21.319 \\
\hline $12 \mathrm{GHz}$ & -17.155 & -19.078 & -18.659 & -18.368 \\
\hline $15 \mathrm{GHz}$ & -14.513 & -14.253 & -13.665 & -14.269 \\
\hline
\end{tabular}

In different angular domains, the RCS curve increases with the increase of frequency. As seen from Figure 6, the scattering curve that the incident frequency is $10 \mathrm{GHz}$ and $12 \mathrm{GHz}$, although the frequency is relatively close (in the same band, that is, within the X band), in the entire angular field, there is still relatively large. Table 1 is RCS mean values in different angles of different incidence frequency, we can see, when grazing, in the $-90^{\circ} \sim-60$ angle domain, the RCS mean values increases with the increase of the incidence frequency. In the- $60^{\circ} \sim-10^{\circ}$ angle domain, when the frequency is respectively $6 \mathrm{GHz}$ and $10 \mathrm{GHz}$, RCS mean value hasn't changed much, it is at about $-22 \mathrm{dBsm}$, however, when the frequency is respectively $12 \mathrm{GHz}$ and $15 \mathrm{GHz}$, which in turn increases $3 \mathrm{~dB}$ and $8 \mathrm{~dB}$ or so, showing the flat increment in the angle domain. For the $\pm 10^{\circ}$ angle domain, because the interference of the scattering of the carrier itself is removed, the corresponding RCS mean is increased with the increase of the frequency. For the $-90^{\circ} \sim 0^{\circ}$ angle domain, RCS mean increases, reflecting the gap RCS increases with the increase of frequency in the overall angular domain.

\section{Conclusion}

From the existing calculation results, with regard to the law of the single straight gap electromagnetic scattering with the width and the incident frequency, the conclusion can be drawn as follows:

(1) When the gap width is less than $1 / 2$ wavelength, gap electromagnetic scattering increases with the increase of the width in the angular domain, when the gap width is more than $1 / 2$ wavelength, gap electromagnetic scattering increases with the increase of the width in the incident angle of $60^{\circ} \sim 60^{\circ}$ range.

(2) For the multi frequency characteristics of the gap, with the increase of the incident frequency, the gap electromagnetic scattering is also increased.

(3) The vector cancellation method can eliminate the interference of slit scattering results from the scattering of the carrier, but the results of the scattering still contain the gap and coupling of gap and edge of carrier. How to eliminate the coupling effect of the edge of the carrier to the gap, making the accuracy of the calculation of the electromagnetic scattering of the gap is a higher problem to be solved.

\section{References}

1. G. Y. He, C. C Lu, J. C. Hong, et a1. The computation and test for RCS(2006)

2. N. Yuan, X. C. Xie, C. H. Liang. Chinese Journal of Radio Science,14, 261 (1999)

3. J. S. Zhu, Y. Yu, B. F. Wang. Journal of Beijing University of Aeronautics and Astronautics, 25, 99 (1999)

4. Z. L. Zhang, W. L. Ni. Chinese Journal of Radio Science, 16,301(2001)

5. J. Z. Chai, X. Gao, X. Q. Liu, J. Q. Ai. Journal of Nanjing University of Aeronautics \& Astronautics, 46,567(2014)

6. THOMAS B. A. SENIOR, KAMAL SARABANDI, JOHN R. NATZKE. IEEE Transactions On Antennas And Propagation, 38,1102 (1990)

7. X. Q. Sheng, Edward K. N. Yung, Chi H. Chan, J. M. Jin, and W. C. Chew. IEEE Transactions On Antennas And Propagation,,48,1153 ( 2000) 
8. A. K. Dominek, H. T. Shamansky, N. Wang. IEEE Transactions On Antennas And Propaga-tion, 37,586 (1989)

9. T. J. Park, S. H. Kang, H. J. Eom. IEEE Transactions On Antennas And Propagation, 42, 112 (1994)

10. P. L. Huang, D. L. Ma, Z. Wu. Journal of Beijing University of Aeronautics and Astronautics, , 27, 293(2001)

11. H. Duan, J. Z. Li, T. Hou. Electronic Measurement Technology, 32, 52 (2009)

12. Z. H. Liu, P. L. Huang, Z. Wu.. Journal Of Hefei University Of Technology(Natural Science), , 32, 1430 (2009)

13. Z. H. Liu, P. L. Huang, X. Gao, Z. Wu. Journal Of Air Force Engineering University(Natural Science Edition), 10, 60 (2009)

14. Z. H. Liu, S. J. Jiang, J. Z. Ji, M. Xu. Journal Of Detection \& Control, 31, 20(2009)

15. G. L. Wang, Z. WU. Aeta Aeronautica et Astro-nautica Sinica, 28, 1116 (2007)

16. Y. X. Li, Q. M. Zhang, Y. L. Long. Chinese Journal of Radio Science, 22, 637 (2007)

17. P. L. Huang, Z. H. Liu. Acta Aeronautica et Ast ronautica Sinica, 29, 675 (2008)

18. X. Gao, Z. H. Liu, Z. Wu. Acta Aeronautica et Ast ronautica Sinica, 29, 1497 (2008)

19. Y. F. Jia. Manufacture Information On Engineering Of China, 2, 59 (2008)

20. J. H. Sang, Z. B. Zhang, S. Wang. Advances In Aeronautical Science And Engineering, 3, 257 (2012) 